\title{
Los pre-congresos como espacios de formación y cualificación docente: la experiencia del V Congreso Popular en Defensa de la Educación Pública. AMP- La Rioja (Argentina)
}

\author{
THE PRE-CONFERENCES AS SPACES FOR TEACHER TRAINING AND QUALIFICATIONS: THE EXPERIENCE \\ OF THE POPULAR CONGRESS V IN DEFENSE OF PUBLIC EDUCATION. AMP-LA RIOJA (ARGENTINA)
}

OS PRÉ-CONGRESSOS COMO ESPAÇOS DE FORMAÇÃO E CUALIFICACIÓN DOCENTE: A EXPERIÊNCIA DO V CONGRESSO POPULAR EM DEFESA DA EDUCAÇÃO PÚBLICA. AMP-A RIOJA (ARGENTINA)

Diana María López Cardona* / dianafilos@gmail.com

\section{Resumen}

El artículo presenta la experiencia de los pre-congresos como espacios de formación docente, para ello toma los realizados en el marco del V Congreso en Defensa de la Educación Pública, llevado a cabo el 8 y 9 de Junio de 2012, por la Asociación de Maestros y Profesores (AMP) de La Rioja, Argentina; se exponen las actividades y reflexiones que surgieron a partir de la asesoría político/pedagógica que la autora desarrolló antes y durante el proceso del Congreso, como una experiencia transformadora que enriquece las prácticas de los/as docentes, la formación y la consolidación de espacios congresales de maestros/as y profesores, sobre la base de la participación activa de los mismos en un proceso de reflexión/acción.

\section{Summary}

The article presents the experience of the pre-congresses as teachers' formation spaces made under The $\mathrm{V}$ Congreso en Defensa de la Educación Pública executed on June $8^{\text {th }} 9^{\text {th }}, 2012$ by the Asociación de Maestros y Profesores (AMP) de La Rioja, Argentina. Here are exposed the activities and reflections raised from the political-pedagogical advise that the author developed before and after the congress process, as a transformative experience that improve the teachers practices, the formation and consolidation of congressional spaces of the teachers, on base of the active participation of themselves in a reflection/action process.

\section{Resumo}

O artigo apresenta a experiência dos pré-congressos como espaços de formação docente, para isso toma os realizados no marco do V Congresso em Defesa da Educação Pública, levado a cabo o 8 e 9 de Junho de 2012, pela Associação de Maestros e Professores (AMP) da Rioja, Argentina; expõem-se as actividades e reflexões que surgiram a partir da assessoria político/pedagógica que a autora desenvolveu dantes e durante o processo do Congresso, como uma experiência transformadora que enriquece as práticas de os/as docentes, a formação e a consolidación de espaços congresales de maestros/as e professores, sobre a base da participação activa dos mesmos num processo de reflexão/acção.

\section{Palabras clave}

Congresos de maestros, procesos de formación político/ pedagógica, sistematización de experiencias, consolidación de organizaciones de maestros/as y profesores.

\section{Keywords}

Congresses of teachers, processes of political-pedagogical formation, systematization of experiences, teachers' organizations' consolidation.

\section{Palavras chave}

Congressos de maestros, processos de formação político/pedagógica, sistematización de experiências, consolidación de organizações de maestros/as e professores.

* Filósofa de la Universidad Nacional de Colombia y Magister en Educación de la Universidad Pedagógica Nacional. Investigadora y formadora de maestros. Estudiante del Doctorado en la Facultad de Filosofía y Letras de la Universidad de Buenos Aires - UBA. Actualmente Asesora Pedagógica de la AMP, La Rioja (Argentina).

Fecha de recepción: 6 de julio de 2012 / Fecha de aprobación: 18 de octubre de 2012 


\section{Introducción}

La Asociación de Maestros y Profesores de La Rioja Argentina es, en la actualidad, uno de los sindicatos de maestros y profesores más fuertes y consolidados del país; cuenta con una historia de lucha que se remonta hasta los años 20, época en la que se crearon las primeras organizaciones sindicales de educadores $y$, hacia los años 70, llega a consolidarse la forma en que es conocida hasta la actualidad (Schulman, 2002, p. 3).

Desde el año 2002, la AMP (como se le conoce al Sindicato), viene realizando Congresos Populares en Defensa de la Educación Pública, contando siempre con gran impacto en el gremio docente por la magnitud de estos encuentros y la importancia de sus declaratorias, alusivas siempre a la defensa del Derecho a la Educación, la construcción de política pública participativa y un elemento central en sus reflexiones: la unidad latinoamericana.

En su penúltimo Congreso, el IV, realizado durante el 2010, dedicado al Bicentenario de la Independencia, la AMP definió como línea de acción para el V Congreso la construcción del Movimiento Pedagógico Latinoamericano:

Queremos que el V Congreso encuentre una CTA y una CTERA fortalecidas, que contribuyan a la construcción de un Movimiento Pedagógico que revolucione a la educación, que transforme para mejor a nuestras organizaciones sindicales, que pueda influir en las políticas públicas y las políticas educativas en particular. Y que permita contribuir a transformar las instituciones educativas, el trabajo docente, la relación pedagógica. (De Leonardi, 2010, p. 1).

En consonancia con los lineamientos, y en construcción conjunta con la Confederación de Trabajadores de la Educación de la República Argentina (CTERA), afiliada a la Central de Trabajadores de la Argentina (CTA), la AMP ha iniciado la consolidación del Movimiento Pedagógico Latinoamericano, una iniciativa de la Internacional de la Educación, que realizó su primer encuentro en Bogotá D.C. durante el mes de Diciembre de 2011 y donde se emitió una declaración oficial del Movimiento desde la perspectiva de construir reflexiones, investigaciones y acciones conjuntas por parte de los maestros y profesores organizados de la región.
Recuperar el legado de los pedagogos nuestroamericanos, reconocer el contexto actual de nuestros países en el tema político/educativo, defender el carácter público de la Educación e impulsar la unidad del movimiento de maestros y profesoral de la región (MPL, 2011, p. 1), fueron entre otras cuestiones, las que se definieron a partir de los acuerdos establecidos en el debate, que utilizaron los siguientes ejes de trabajo:

1. Hacia un Movimiento Pedagógico Latinoamericano.

2. El papel del Estado en la garantía de la educación como derecho.

3. Política Pública Educativa.

4. El currículo.

5. Calidad de la evaluación.

6. Formación y valoración de las/os trabajadores de la educación.

Los ejes permitieron el desarrollo de unas relatorías y el encuentro emitió una declaratoria oficial; todo el material fue retomado por el proceso del V Congreso de la AMP en La Rioja y los ejes temáticos fueron transversales para todas las reflexiones, disertaciones y exposiciones que se realizaron durante el mismo.

Es así que el V Congreso, desde su propuesta inicial, retomó las banderas de la construcción del Movimiento Pedagógico Latinoamericano y se lanzó a realizar un proceso de reflexión y consolidación a partir de las prácticas de los maestros/as de la Provincia, con ejercicios previos denominados Pre-Congresos, donde se reflexionó acerca de la iniciativa de MPL y se dieron pautas para la sistematización de las prácticas de los maestros y profesores de La Rioja, que luego fueron expuestas en el $\mathrm{V}$ Congreso como resultado del ejercicio previo y que se presentan compiladas en las memorias del mismo.

Para realizar los Pre-Congresos en toda la Provincia, la AMP se apoyó en la asesoría pedagógico/política de quien escribe y en la oficina de capacitación del sindicato, con la que iniciamos el proceso de acompañamiento y desarrollo de la propuesta. 


\section{Objetivos y metas}

Se definieron 2 objetivos principales dentro del proceso Pre-congresal, teniendo como perspectiva conseguir el objetivo general: la consolidación del proceso de $\mathrm{V}$ Congreso que se realizaría en el mes de Junio de 2012, ellos fueron:

- Reconocer las producciones de los maestros/as de la región, a partir de la elaboración de ponencias que sistematicen experiencias o reflexionen sobre alguno de los ejes temáticos propuestos y coincidentes con MPL

Como estrategia de concreción del objetivo realizamos el taller de Lecto-Escritura a partir de cuatro momentos: 3 actividades en los Pre-congresos, 1 asesoría In situ y varias asesorías virtuales, hasta la elaboración final de las ponencias y sus presentaciones en el Congreso.

- Reflexionar y enriquecer las propuestas de Movimiento Pedagógico Latinoamericano a partir de los documentos producidos por el movimiento.

Se utilizó como estrategia de concreción la lectura, análisis y enriquecimiento de los documentos producidos por MPL en 3 momentos: Presentación de los ejes temáticos compartidos; lectura y análisis de la Declaración de Bogotá; y lectura, análisis y desarrollo de propuestas para el MPL a partir de las relatorías del 1 er Encuentro de MPL en Bogotá.

\section{Metodología: los Pre-Congresos}

Para desarrollar los objetivos propuestos se realizó una convocatoria abierta a todos/as las maestras y profesores de la Provincia, sin importar si estaban o no afiliados a la AMP, luego se distribuyó la población en 8 ciudades a las que nos trasladábamos para hacer el trabajo pre-congresal; el equipo que viajó estaba compuesto por: Ingrid Mercado, responsable de la oficina de capacitación de la AMP; Nilda Chumbita, Secretaria del Interior, y Santiago Cortés, responsable de la logística de los Pre-congresos y delegado de la AMP. Se realizaron 3 Pre-congresos, 1 por mes, de forma simultánea en las 8 ciudades: Aimogasta, Chamical, Chepes, Olta, Ulapes, Chilecito, Villa Unión y la ciudad de La Rioja (todas dentro de la provincia de La Rioja-Argentina).
Al primer Pre-congreso se presentaron alrededor de 600 maestros/as y profesores, con una asistencia de 40 a 50 participantes por ciudad, inquietos y a la expectativa frente a la propuesta que les Ilevaba el sindicato, esta vez con la particularidad de tener estos encuentros y con una asesoría externa puntual. Allí se presentó la propuesta del proceso y por qué entendíamos al V Congreso como un proceso de construcción colectiva, que iniciaba en este primer momento, requería del concurso de los maestros/as de la provincia, y que partiría de sus prácticas y reflexiones sobre los 6 ejes temáticos que fueron transversales a toda la propuesta y que en el congreso tuvieron su lugar de privilegio.

Estos seis ejes temáticos fueron los mismos que se trabajaron en el primer encuentro de MPL; además realizamos el primer taller de lecto-escritura, a manera de introducción y como agente motivacional; a continuación se solicitó a los/as participantes que escogieran su tema de trabajo para la elaboración de las ponencias que se presentarían en el Congreso, en este momento se realizaron 110 propuestas para la sistematización y reflexión sobre los ejes temáticos.

El segundo Pre-congreso se realizó durante el mes de Marzo; acudieron aproximadamente 450 maestros/as, hubo una reducción en la asistencia en relación con el primer pre-congreso debido a que ya se habían iniciado las clases y algunos de los directores/as de escuela, a pesar de tener conocimiento de la actividad, sólo permitieron la participación de los delegados de cada escuela, teniendo el aval los demás para participar; sin embargo, fue significativo el número y en especial el interés por el trabajo propuesto. En esta ocasión también se realizó el taller de lecto-escritura, dando algunas herramientas para la pre-escritura de las ponencias y se dio lectura a la Declaración del MPL.

El tercer Pre-congreso se realizó en el mes de Abril y contó con la asistencia promedio del segundo Pre-congreso. Se realizó el tercer taller de lecto-escritura, en el que se dieron algunas herramientas para la elaboración de la ponencia; además, se hizo la lectura en grupo de las relatorías de los ejes de MPL, de acuerdo al interés de los compañeros frente a las temáticas ofrecidas, y se elaboró una ficha con aportes al MPL.

El cuarto encuentro fue realizado durante el mes de Mayo y no tuvo carácter de Pre-congreso, sino que giró alrededor de una asesoría puntual a cada grupo y tuvo como énfasis la elaboración escrita de las ponencias y 
sus presentaciones para la exposición en el V Congreso. Allí se definieron un poco más de 30 ponencias, a partir de las cuales se continuó con la asesoría virtual hasta concretar el producto final.

Una semana antes del Congreso también se realizó un Pre-Congreso en la ciudad de Buenos Aires, en el Centro Cultural de la Cooperación Floreal Gorini, con la asistencia de maestros/as y de profesores miembros del Centro, del grupo Rayuela, del Sindicato de la Provincia de BsAs, SUTEBA y del sindicato de profesores de la ciudad, UTE; se contó con la asistencia de aproximadamente 70 participantes que disertaron sobre las relatorías de Movimiento Pedagógico y a quienes se les dio toda la información acerca del proceso del Congreso en La Rioja y de su asistencia al mismo. Este grupo acordó volver a reunirse, con el ánimo de continuar reflexionando acerca de cómo construir y consolidar la propuesta de Movimiento Pedagógico en la Ciudad y la Provincia de BsAs.

\section{El Taller de lecto-escritura como proceso de sistematización/reflexión/acción}

Los talleres de lecto-escritura, realizados durante el proceso pre-congresal, fueron diseñados sobre la base de la experiencia de otros procesos de sistematización llevados a cabo en Bogotá-Colombia, especialmente con los aportes de experiencias en formación político-sindical y en formación de docentes y directivos docentes; siempre teniendo en cuenta la necesidad e importancia de avanzar en la producción textual a partir de lecturas y escrituras motivacionales que permitan a los docentes reconocer en esta dupla, una posibilidad para reflexionar y enriquecer su práctica, y a la vez rescatar la importancia de su trabajo cotidiano que, aunque poco valorado, tiene un fuerza y una relevancia innegable en sus comunidades.

La lecto-escritura es un proceso dual que no se da por separado: no hay lectura sin escritura, de la misma manera que no hay escritura sin lectura. La lectura inicial, que se puede hacer como un primer acercamiento al texto, requiere, igual que cualquier otro tipo de lectura más profunda, de la escritura que simula el diálogo del lector con el autor. No es cierto que se lee todo y luego se escribe; al contrario, desde el comienzo de la lectura se inicia un diálogo con el autor que sugiere subrayar el texto, hacer notas al pie, al borde, interrogaciones, exclamaciones y todo tipo de símbolos que ya son una escritura inicial.

De la misma manera, la escritura es un ejercicio inspirado en la lectura, en el diálogo con otros autores, en la reflexión colectiva que no es otra cosa que la lectura espontánea de cada persona. Se trata de "pasar de la lectura de la palabra a la lectura del mundo", dice Freire con fuerza cuando se refiere al salto epistemológico que debe sugerir el proceso de lectura y escritura (Freire, 1983, p. 25). La lectura inicial será la de la palabra, pero cuando se reflexiona se toma posición y se escribe sobre la experiencia directa; estoy haciendo una lectura del mundo, lo que significa que se ha trascendido el proceso a otro nivel de conocimiento que no se da en la lectura inicial ni en la lectura desprevenida.

Ese paso a la lectura del mundo, que es la realidad en que habita el maestro en su cotidianidad, permite reconocer elementos de su trabajo que no se ven a simple vista. Esa reflexión sobre su día a día hace que el maestro encuentre, en su cotidianidad, elementos para compartir y aportar, así como situaciones y puntos para transformar en su práctica y en la vida de la escuela (Freire, 1993, pp. 23-24). De esta manera, la lecto-escritura, como proceso de concienciación, es transcendente y transformadora, porque reconoce a los sujetos en sus imaginarios y relaciones, a su vez que les permite ver cuáles son las cosas que les gustaría cambiar de esa realidad.

Este momento motivacional y de sistematización de su práctica es inicial, por lo que se hace necesario continuar el ejercicio en la perspectiva de ir pasando por niveles de concienciación y de transformación, hasta dar sentido a los cambios y construcciones colectivas para el mejoramiento de la escuela y de sus múltiples complejidades. En ese sentido, se requiere pasar a procesos cada vez más complejos de sistematización hasta la construcción de proyectos propios y autónomos.

Con esto en mente, y tomando como referentes las reflexiones de algunos autores que consideran la escritura una forma de investigación y de gran importancia en la labor docente (Vásquez, 2008, p. 5), se construyeron cinco momentos de lectura y escritura para el desarrollo del proceso del Congreso: 


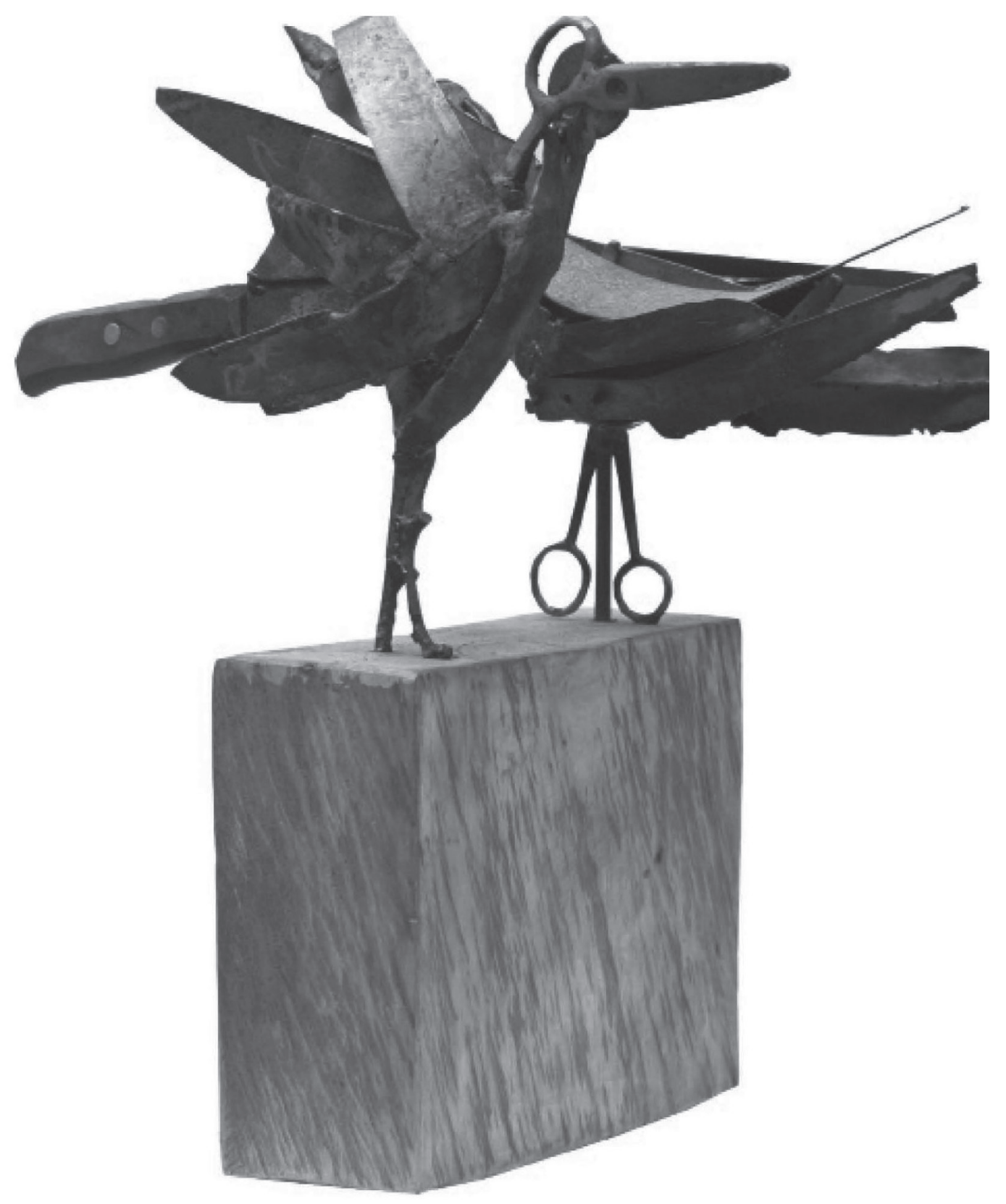

Artista: Felipe Ruiz

Dimensiones: 40x40x20 cm

Tecnica: Escultura en metal

Año: 2010 
a) Lectura y escritura motivacional: diferencia entre el discurso oral y el escrito

El taller se realizó en el Primer Pre-Congreso, allí se dio lectura al texto del profesor Fernando Vásquez Rodríguez: Opus Tessellatum. Un Mosaico alrededor de la Escritura, en el cual da cuenta de las diferencias entre el discurso oral y el escrito; sin decir que son antagónicos o ver a uno como mejor que el otro, afirma que son distintos en sus finalidades y composiciones. Además, muestra la importancia de la escritura en la labor docente y da algunas pistas para comenzar a registrar las ideas que nos asaltan en este ejercicio cotidiano (Vásquez, 2008, p. 135).

Luego de la lectura realizamos un ejercicio escritural motivacional: cada uno de los participantes debía escribir el primer recuerdo que tenía de su vida (López y Otros, 2007, p. 83); un trabajo realizado por la profesora Yolanda González de Zuleta, de la Universidad del Valle, en sus talleres de lecto-escritura y que he incluido en los procesos de formación que realizo con docentes y trabajadores. Esta práctica da unos resultados impresionantes en cuanto al despliegue de creatividad y, en algunos casos, de una realidad cruda que demuestra la profundidad de los pensamientos de sus escritores.

A partir de este ejercicio surgió otro complementario elaborado por la profesora de artes Susana Andrada, delegada de la AMP; consistió en dibujar a partir de los mensajes de los recuerdos. El trabajo con los recuerdos y los dibujos fue expuesto en el salón principal, donde se desarrolló el $\mathrm{V}$ Congreso, a manera de registro de esta actividad que resultó ser artística, creadora e inspiradora; aquí una pequeña muestra de los resultados del taller:

\section{El extraño de pelo largo}

No sé realmente si es mi primer recuerdo, pero sí estoy seguro de que es el más fuerte: el primer día en el jardín de infantes; es un poco gracioso, pero jamás me dio vergüenza contarlo.

Con seis años cumplidos, aún no me habían cortado el cabello, fue por una promesa que mis padres habían realizado. Recuerdo claramente que la maestra me hizo sentar con las niñas, no recuerdo bien en qué o lo que hicimos, pero a la hora de jugar me fui con los varones a patear una pelota; fue ahí donde la distraída maestra se dio cuenta de que yo en realidad era un varón.
Lo que sucedió fue que quién escribió la lista de alumnos, escribió mal mi nombre, puso Rafaela en lugar de Rafael.

\section{Rafael}

El siguiente momento lecto-escritural de este taller fue la propuesta de elegir el tema a presentar en el V Congreso. Este ejercicio se realizó en grupo, completando una ficha que solicitaba los datos básicos de los participantes, el tema y una descripción inicial del mismo, así como una posibilidad del eje en el cual se podría presentar. Con estas fichas fue posible tener un registro del proceso de todas las propuestas que Ilegaron a consolidarse como ponencias y que fueron presentadas; en este primer momento se realizaron 110 propuestas.

\section{b) Momentos de la escritura: macro-estructura y elaboración de ensayos}

Este taller se realizó en el Segundo Pre-congreso, comenzó con una exposición acerca de las herramientas previas a la escritura, es decir de la importancia de los ejercicios pre-escriturales, pues nadie comienza a escribir en frío; lo cierto es que ni los más avezados escritores terminan sus obras en un único momento escritural, su trabajo pasa por diferentes momentos de pre- escritura y pos-escritura que luego se complementan con los editores y los correctores de estilo. En ese sentido, es importante reconocer a la escritura como un ejercicio que requiere de disciplina, constancia y motivación, como cualquier otro trabajo.

Para este momento se tuvieron en cuenta los aportes del profesor Daniel Cassany y la primera parte de su libro La cocina de la escritura, donde muestra cómo los primeros rasgos de la escritura se dan a partir de los trazos de unos esquemas básicos que se van convirtiendo paulatinamente en esquemas cada vez más complejos, hasta llegar a los mapas conceptuales y a la macro-estructura del texto. Estos ejercicios de elaboración de esquemas están relacionados con la manera como comenzamos nuestro proceso lecto-escritural cuando somos niños, a partir del dibujo; ejercicio que poco a poco va siendo desplazado y hasta olvidado (Cassany, 1995, p. 28).

En este momento recuperamos el dibujo para comenzar a trazar la pre-escritura de la ponencia; para ello se propuso retomar los temas escogidos en el primer Pre- 
congreso y convertirlos en esquemas de lo que sería luego nuestro ejercicio escritural. Se entregó un formato para volver a completar los datos básicos, arriesgar un título, distinguir el tema y hacer el esquema. Si todavía era muy difícil realizarlo se sugirió que detrás de la ficha se realizara una Iluvia de ideas iniciales para luego convertirlas en un dibujo que mostrara cuál es el tema central o la idea principal sobre la que iba a girar el resto del texto y desplegar, a partir de allí, las otras ideas. Aquí se presentaron 83 propuestas que daban cuenta del esquema inicial de la ponencia.

\section{c) Elaboración de ponencias: pautas para construir ensayos}

Este taller se realizó en el 3er Pre-congreso y tuvo como objetivo ordenar el esquema realizado, previo a la escritura, en lo que podrían ser el título y los subtítulos de la ponencia. Así que inicialmente se proporcionaron algunas pautas para la elaboración de ensayos, basadas en el texto de clase del profesor Carlos Medina Gallego: Pautas para la elaboración de ensayos, que utiliza con sus estudiantes de Ciencias Políticas de la Universidad Nacional y que recoge de manera clara y sucinta los elementos de un ensayo y cuál es su objetivo (Medina, 2005, p. 3).

En esta ocasión tuvimos el consolidado de 66 ejercicios donde se ubicaba el título y subtítulo o subtítulos de la ponencia, que luego sería complementada con frases y párrafos que dieran cuenta de los subtemas decididos desde la elaboración del esquema y que ahora cobraban forma en los subtítulos. En algunos de ellos hubo cambios del primer ejercicio a este, otros cambiaron su contenido; mientras que la mayoría tuvieron transformaciones de forma y composición, es decir, lo que inicialmente se veía como importante pasó a un segundo plano y viceversa. Los participantes encontraron un orden que luego se evidenció en la escritura de las ponencias.

\section{d) Asesorías In situ y virtuales: escritura y elaboración de presentaciones para la exposición en el $V$ Congreso}

En este momento del proceso regresamos a las ciudades y se realizó una asesoría in situ con los grupos que ya estaban escribiendo, tomando grupo por grupo y considerando la escritura y la presentación de la ponencia, se mostró la importancia de ubicar el contexto de la experiencia y la necesidad de hacer énfasis en las ideas que consideraban como más fuertes dentro de la propuesta. Teniendo en cuenta que la mayoría de estas/os maestros no habían presentado una ponencia, era importante explicar muy bien en qué consistía el ejercicio y qué se podría presentar. Sin embargo, no hubo una selección de nuestra parte, fue realizada por ellos durante el proceso, cuando decidieron abandonar el ejercicio o continuar en él.

Las asesorías continuaron de manera virtual con la mayoría de quienes expusieron, aunque hubo quienes presentaron la ponencia escrita y no la socializaron en el Congreso por diversos motivos, así es que tuvimos 30 ponencias escritas y 27 exposiciones, lo que evidencia un grado de compromiso muy interesante el de las/os maestros a la hora de asumir su presentación.

\section{e) Presentación de las ponencias en el V Congreso}

El sábado 9 de Junio de 2012, en la Escuela de Comercio $\mathrm{N}^{\circ} 1$ de la ciudad de La Rioja, abrimos la presentación de las ponencias con esta explicación sobre lo que fueron los ejercicios pre-congresales y luego los maestros/as se presentaron por ejes temáticos. En su mayoría realizaron presentaciones powerpoint, a pesar de la resistencia de algunos a utilizar estas ayudas. Al final se decidieron por las presentaciones e incluso la realización de pequeños videos que daban cuenta de sus experiencias en las escuelas de toda la Provincia. Más adelante se encontrarán las ponencias escritas por los maestros/as, distribuidas por los ejes temáticos donde las expusieron.

\section{El V Congreso}

EI V Congreso en Defensa de la Educación Pública se llevó a cabo pensando en la construcción del Movimiento Pedagógico Latinoamericano, se realizó en la ciudad de La Rioja los días 8 y 9 de Junio de 2012 y contó con la presencia de personalidades latinoamericanas de la educación y en especial de las organizaciones sindicales de educadores de la región; entre ellos se encontraban:

- Guillermo Scherping. Miembro del Colegio de Profesores de Chile y de la Central de Trabajadores del mismo país. 
- Combertty Rodríguez. Miembro de la Internacional de la Educación y parte de la Organización Sindical de Maestros de Costa Rica.

- Orlando Pérez. Secretario General del Sindicato de Maestros y Profesores de Venezuela SINAFUM.

- Mirna Sojo. Miembro de la Escuela Social Rodrigueana del Estado de Aragua de Venezuela.

- Carlos Lanz. Asesor del Ministerio para el Poder Popular de la Educación de Venezuela.

- Ramón Cardona. Secretario para América Latina de la Federación Sindical Mundial, FSM.

- $\quad$ Edith Santos. Miembro de AELAC de Cuba.

- Stella Maldonado. Secretaria General de la Confederación de Trabajadores de la Educación de Argentina, CTERA.

- Pablo Imen. Vicedirector del Programa Latinomericano de Educación a Distacia, PLED, y Coordinador del Departamento de Investigaciones del Centro Cultural Floreal Gorini, CCC. Argentina.

- Rogelio de Leonardi. Secretario General de la Asociación de Maestros y Profesores de La Rioja, AMP.

Con este nutrido grupo de invitados y otros tantos que llegaron de otras provincias de todo el país, se reflejó no sólo la organización del sindicato, sino la importancia de este proceso para el desarrollo de los objetivos conjuntos de las organizaciones de maestros del país y la región. Desde la perspectiva más latinoamericana, cada uno de los invitados reflexionó sobre la importancia de la defensa de la educación pública; la necesidad del reconocimiento de la labor docente; el momento tan especial de integración que vive la región, y la necesidad de seguir trabajando en favor de la unidad de los movimientos sociales, sindicales $y$, en particular, del movimiento de maestros y profesores. Cada una de sus ponencias, da cuenta de la complejidad y riqueza de los elementos que se plantearon y propusieron a los más de 1.200 docentes que asistieron durante el Congreso.

La exposición del segundo día, que cubría la experiencia de pre-congresos y las exposiciones de las más de 30 ponencias que se realizaron durante este proceso, fue quizá el elemento más innovador de la presentación; ya que los Congresos siempre son eventos que inician y terminan en ellos mismos y en pocas ocasiones son los/as docentes los que se convierten en sus protagonistas. Esto ya demuestra un cambio en la perspectiva de construcción y presenta un nuevo camino para la construcción de un movimiento docente que tenga en cuenta la voz de sus protagonistas, sus historias, sus angustias, sus procesos y sus logros.

\section{Los espacios de reflexión y aportes al Movimiento Pedagógico Latinoamericano}

Durante los Pre-congresos y como una definición inicial de la AMP, la convocatoria se realizó con la perspectiva de la construcción del Movimiento Pedagógico Latinoamericano, así que en el Primer encuentro explicamos en detalle las líneas de acción convergentes con el mismo (MPL, 2011, p. 2):

- Hacia la política pública. Exigir al Estado el cumplimiento de sus responsabilidades como garante del Derecho a la Educación, asumiendo su compromiso con el financiamiento de la misma, pero al mismo tiempo el desarrollo de políticas que aseguren una educación digna que debe construirse a partir del diálogo de saberes, con un papel protagónico de los docentes y la comunidad educativa, superando la lógica jerárquica y tecnocrática. Avanzar en un modelo de construcción participativa del currículo y en la sostenida implementación de mecanismos de trabajo colectivos entre docentes.

- Hacia la comunidad territorial. Supone nuevos vínculos de la institución escolar con la comunidad de la que es parte, avanzando en la noción de escuela como centro del quehacer comunitario y de comunidad como centro del quehacer educativo. Una educación para la vida reclama salir del aula y las paredes para imbricar el proceso pedagógico con la realidad del territorio.

- Hacia la propia organización sindical. Desplegar acciones de reorganización y de formación de los dirigentes sindicales, los delegados y quienes están más comprometidos con la defensa de la educación pública, adecuando la dinámica sindical a los requerimientos del Movimiento Pedagógico, sin olvidar sus funciones históricas, ligadas a la defensa de los derechos de los trabajadores 
- Hacia la construcción de un modelo pedagógico emancipador. El sindicato debe promover un fuerte trabajo en las instituciones educativas, que sea fruto de una participación protagónica de los docentes en diálogo con otros interesados.

Estas líneas de acción han sido el motor para desarrollar las reflexiones, sistematizaciones y demás ejercicios pedagógicos de consolidación de propuestas, encaminadas al V Congreso, y a la construcción de Movimiento Pedagógico en nuestra región y el país; se desarrollaron a través de 6 ejes temáticos asumidos como parte de la propuesta del MPL:

- Eje 1: Características del Movimiento Pedagógico Latinoamericano: integración regional con sentido antiimperialista, multicultural y de respeto por la diversidad.

- $\quad$ Eje 2: Política Educativa: en la que se priorice la educación como derecho y se afiance la educación pública, democrática y con justicia social.

- Eje 3: El papel del Estado: como garante del derecho social a una educación pública de calidad, digna y para todos y todas.

- Eje 4: Calidad de la educación: alrededor del desarrollo de condiciones favorables para las comunidades educativas de acuerdo a sus realidades y necesidades concretas, factores de reconocimiento y posibilidad de realización de la labor educativa; currículo de construcción colectiva y evaluación integral, donde se prioricen los procesos y desarrollos de las comunidades.

- Eje 5: Currículo: como una construcción colectiva, que atienda a las necesidades y objetivos propios de las comunidades, con sentido global y local.

- Eje 6: Formación y valoración de las trabajadoras y trabajadores de la educación: condiciones para que sea posible el trabajo docente colectivo e interdisciplinario, recuperando el control de las trabajadoras y trabajadores sobre el proceso educativo.

Fue a partir de estos ejes que los/as participantes se distribuyeron para trabajar tanto los documentos del $\mathrm{MPL}$, como las ponencias que luego se presentarían en el Congreso.
Durante el segundo encuentro se realizó la lectura de la Declaración Oficial del MPL, denominada Declaración de Bogotá y, a partir de allí, se realizaron disertaciones por grupos y propuestas encaminadas a enriquecer el documento. Mientras que en el tercer encuentro se leyeron las relatorías de los ejes temáticos abordados por $M P L$, y se hicieron aportes a las mismas, pensando en relación con el trabajo particular de los/as participantes en la Provincia de La Rioja y en Argentina como un país convocante a MPL.

De todas estas reflexiones surgieron aportes recogidos en una ficha con consignas que luego se tuvieron en cuenta para la elaboración de la declaración final del $\checkmark$ Congreso y en las que se les consultaba acerca de cuáles serían los elementos que considera relevantes en la construcción de MPL y que no están consignados en la Declaración de Bogotá, cuáles serían las actividades que se le sugerirían realizar y cuáles se podrían hacer desde sus lugares de trabajo.

\section{Resultados y continuidades}

Dentro de los más variados resultados y posibilidades de continuidad del proceso, hemos definido como más relevantes los siguientes:

- 30 ponencias escritas con sus exposiciones para socializar: compiladas en las memorias y que se convierten en el primer insumo a analizar para continuar enriqueciendo las prácticas de los/as maestras que se vincularon a este proceso, y que seguro le darán continuidad.

- Un documento oficial de aportes al Movimiento Pedagógico Latinoamericano: en la declaración final del $V$ Congreso, quedaron plasmadas las reflexiones y aportes de los/as participantes del proceso como aporte al inicio de la construcción de MPL en la región y en la Argentina.

- Inicio y continuación de un proceso de formación política, sindical, pedagógica y cultural: teniendo en cuenta los avances y resultados significativos del proceso, la AMP decidió continuar con lo iniciado a través de la consolidación de un Programa de Formación Permanente, que inicialmente estará a cargo de la Asesoría Pedagógica del Congreso. 
- Procesos organizativos en las regiones: con el avance de la AMP en la consolidación del Movimiento Pedagógico Latinoamericano, se han venido impulsando otros procesos con organizaciones afiliadas a CTERA y a otras organizaciones de maestros/as y profesores que, durante este año y el próximo, realizarán actividades encaminadas a fortalecer este proceso de construcción.

- Procesos de constitución de equipos de maestros/ as de la Provincia (Mapeo): se realizó un rastreo inicial de grupos de maestros de toda la Provincia, que está por ser sistematizado y actualizado para consolidar un cuadro que permita tener la información necesaria para próximas actividades del MPL.

- Fortalecimiento de la AMP: Este proceso sin duda sirvió para fortalecer el sindicato, que además contó con nuevos docentes afiliados, permitiendo de esta manera el reconocimiento de la importancia de actividades como las desarrolladas, que van más allá de las reivindicaciones laborales y afianzan procesos de formación de sus afiliados y de fortalecimiento de la organización.

- Congreso: la realización del V Congreso y la construcción del próximo: el VI Congreso al 2014: Pedagogías Emancipadoras de Nuestra América.

\section{Referencias}

Cassany, D. (1995). La cocina de la escritura. Barcelona: Anagrama.

De Leonardi, R. (2010). Carta del IV Congreso Popular en Defensa de la Educación Pública. La Rioja: AMP.

Freire, P. (1983). La Educación como práctica de la libertad. Madrid: Siglo XXI.

Freire, P. (1993). Política y Educación. Madrid: Siglo XXI.

López, D. Et al. (2005). Hacia una conciencia transformadora: reflexiones sobre una experiencia de formación sindical en Colombia. Bogotá: ACCD.

Medina, C. (2005). Guía para la elaboración de Ensayos: documento de formación básica. Bogotá: UNAL. (Material de clase).

Movimiento Pedagógico Latinoamericano. (2011). Declaración de Bogotá. Bogotá.

Schulman, J. (2002). La Rioja que Resiste. La Rioja: AMP.

Vásquez R. F. (2008). Escritores en su tinta. Bogotá: Kimprex.

Vásquez R. F. (2008a). "La Escritura y su Utilidad en la docencia". Actualidades Pedagógicas. No 51. Bogotá: U. de la Salle.

\section{Diálogo del conocimiento}

Los movimientos populares no han desaparecido. Argentina vuelve a dar ejemplo al convocar y reunir las voluntades de la Asociación de Maestros y Profesores, AMP, de la Rioja. Utilizando como estrategia la realización de pre-congresos, y como metodología talleres de escritura y lectura continúan con el legado de Freire y de Mario Kaplún para articular tres de sus propósitos centrales a un trabajo conjunto, sistemático, concienciado y comunicado: Derecho a la educación, la construcción de política pública participativa, y la unidad latinoamericana a través de un Movimiento pedagógico.

Podemos entender que estos nuevos sujetos políticos que escriben sobre sus experiencias, que siempre es una toma de posición frente al mundo; además, que visibilizan sus voces y se retroalimentan en el pre-congreso, el cual les sirve como espacio de formación; buscan en últimas incidir positivamente en la política nacional del país, así como en la transformación inmediata de sus contextos. Así que, tal como lo menciona el artículo, la puesta en marcha de un Movimiento pedagógico, trae consigo la idea de una transformación paulatina, meditada, pertinente, contextualizada, construida desde los saberes de base, la realidad y la experiencia, y sumada a las voluntades de todos quienes trabajan en la construcción de una Latinoamérica con pensamiento propio.

Convocar, encontrar, escribir, releer, sistematizar, disertar, construir, declarar, son verbos que condujeron a la acción concreta durante las 24 sesiones pre-congresales distribuidas en ocho ciudades argentinas que permitieron como trabajo del pre-congreso anudar una nuevo horizonte latinoamericano en cuanto a educación, política pública, el papel del Estado, currículo, calidad de la educación, y visibilizarse a lo largo del V Congreso en Defensa de la Educación Pública realizado en el mes de junio de 2012 en la Rioja, Argentina.

Edilson Silva Liévano 